\title{
ON KAEHLER MANIFOLDS SATISFYING THE AXIOM OF ANTIHOLOMORPHIC 2-SPHERES
}

\author{
MINORU HARADA
}

\begin{abstract}
A Kaehler manifold with the axiom of antiholomorphic 2-spheres is a complex space form.
\end{abstract}

1. Introduction. Let $M$ be a Kaehler manifold with complex structure $J$ and Riemann metric $g$.

By a plane section we mean a 2-dimensional linear subspace of a tangent space. A plane section $\pi$ is called holomorphic (resp. antiholomorphic) if $J \pi=\pi$ (resp. $J \pi$ is perpendicular to $\pi$ ). The sectional curvature for a holomorphic (resp. antiholomorphic) plane section is called holomorphic (resp. antiholomorphic) sectional curvature.

A Kaehler manifold of constant holomorphic sectional curvature is called a complex space form. It is well known that a complex space form has constant antiholomorphic sectional curvature.

Conversely, in their recent paper [1], B. Y: Chen and K. Ogiue proved that a Kaehler manifold with dimension $\geqq 3$ and constant antiholomorphic sectional curvature is a complex space form.

A Kaehler manifold $M$ is said to satisfy the axiom of holomorphic planes (resp. axiom of antihomomorphic planes) if, for each $x \in M$ and each holomorphic (resp. antiholomorphic) plane $\pi \subset T_{x}(M)$, there exists a 2-dimensional totally geodesic submanifold $N$ such that $x \in N$ and $T_{x}(N)=\pi$. I. Mogi and K. Yano [4] proved that a Kaehler manifold with the axiom of holomorphic planes is a complex space form.

Recently, B. Y. Chen and K. Ogiue [1] proved that a Kaehler manifold with dimension $\geqq 3$ and the axiom of antiholomorphic planes is a complex space form.

A Riemannian manifold $M$ of (real) dimension $\geqq 3$ is said to satisfy the axiom of 2-spheres if, for each $x \in M$ and each plane $\pi \subset T_{x}(M)$, there exists a 2-dimensional umbilical submanifold $N$ with parallel mean curvature vector field such that $x \in N$ and $T_{x}(N)=\pi$. D. Leung and K. Nomizu [3] proved that a manifold with this property has constant sectional curvature.

Received by the editors May 23, 1973.

AMS (MOS) subject classifications (1970). Primary 53B35.

Key words and phrases. Kaehler manifold, complex space form, axiom of antiholomorphic 2-spheres.

(c) American Mathematical Society 1974 
Recently, in his paper [2], S. Goldberg introduced the axiom of holomorphic 2-spheres; a Hermitian manifold $M$ is said to satisfy the axiom of holomorphic 2-sphere if, for each $x \in M$ and each holomorphic plane $\pi \subset T_{x}(M)$, there exists a 2-dimensional umbilical submanifold $N$ with parallel mean curvature vector field such that $x \in N$ and $T_{x}(N)=\pi$. He proved that a Kaehler manifold satisfying the axiom of holomorphic 2-spheres has constant holomorphic sectional curvature.

A Kaehler manifold is said to satisfy the axiom of antiholomorphic 2-spheres if, for each $x \in M$ and each antiholomorphic plane $\pi \subset T_{x}(M)$, there exists a 2-dimensional umbilical submanifold $N$ with parallel mean curvature vector field such that $x \in N$ and $T_{x}(N)=\pi$.

We shall prove the following theorem in this paper.

THEOREM. Let $M$ be a Kaehler manifold. If $M$ satisfies the axiom of antiholomorphic 2-spheres and if $\operatorname{dim} M \geqq 3$, then $M$ is a complex space form.

The author expresses his hearty thanks to Professor K. Ogiue for his valuable suggestions and encouragement.

2. Preliminaries. Let $M$ be a Kaehler manifold with complex structure $J$ and Riemann metric $g$. We denote by $R$ the curvature tensor field of $M$. Then we have

$$
\begin{gathered}
R(J X, J Y)=R(X, Y), \\
R(X, Y) J Z=J R(X, Y) Z .
\end{gathered}
$$

Let $K(X, Y)$ be the sectional curvature of $M$ determined by orthonormal vectors $X$ and $Y$. Then we have

$$
\begin{aligned}
& K(J X, J Y)=K(X, Y), \\
& K(X, J Y)=K(J X, Y) .
\end{aligned}
$$

The following is easily seen.

Orthonormal vectors $X$ and $Y$ span an antiholomorphic section if and only if $X, Y$ and $J X$ are orthonormal.

Let $N$ be a submanifold of $M$ and let $\tilde{\nabla}$ and $\nabla$ be the covariant differentiations on $M$ and $N$ respectively. Then the second fundamental form $\sigma$ of the immersion is defined by

$$
\sigma(X, Y)=\tilde{\nabla}_{X} Y-\nabla_{X} Y,
$$

where $X$ and $Y$ are vector fields tangent to $N . \sigma$ is a normal bundle valued 
symmetric 2 -form on $N$. For a vector field $\xi$ normal to $N$ we write $\tilde{\nabla}_{X} \xi=$ $-A_{\xi} X+D_{X} \xi$, where $-A_{\xi} X$ (resp. $D_{X} \xi$ ) denotes the tangential (resp. normal) component of $\tilde{\nabla}_{X} \xi$. The tensor fields $\sigma$ and $A_{\xi}$ are related by

$$
g(\sigma(X, Y), \xi)=g\left(A_{\xi} X, Y\right) .
$$

Since $R(X, Y) \xi=\tilde{\nabla}_{X} \tilde{\nabla}_{Y} \xi-\tilde{\nabla}_{Y} \tilde{\nabla}_{X} \xi-\tilde{\nabla}_{[X, Y]} \xi$, we can obtain

$$
\begin{aligned}
R(X, Y) \xi= & \left(\nabla_{Y^{\prime}} A_{\xi}\right) X-\left(\nabla_{X} A_{\xi}\right) Y \\
& +A_{D_{X^{\xi}}} Y-A_{D_{X^{\xi}}} X \text { (modulo normal component). }
\end{aligned}
$$

The mean curvature normal $H$ of $N$ in $M$ is defined by the relation

$$
\text { trace } A_{\xi}=2 g(\xi, H),
$$

for all $\xi$ normal to $N$. It is called parallel (in the normal bundle) if $D H=0$. The surface $N$ is umbilical in $M$ if $\sigma(X, Y)=g(X, Y) H$, i.e., if

$$
A_{\xi}=g(\xi, H) I=\frac{1}{2}\left(\operatorname{trace} A_{\xi}\right) I,
$$

where $I$ is the identity transformation.

An umbilical submanifold is totally geodesic if $H$ vanishes.

3. Proof of theorem. Let $x$ be an arbitrary point of $M$ and let $X$ and $Y$ be arbitrary orthonormal vectors in $T_{x}(M)$ which span an antiholomorphic section $\pi$. Then, there is an umbilical submanifold $N$ with parallel mean curvature normal $H$ such that $x \in N$ and $T_{x}(N)=\pi$. Let $U$ be a normal neighborhood of $x$ in $N$ and for each $y \in U$ let $\xi_{y}$ be the normal to $N$ at $y$ parallel (with respect to $D$ ) to $J X$ along the geodesic in $U$ from $x$ to $y$. Along each such geodesic, $g(\xi, H)$ is a constant $c$, since $\xi$ and $H$ are parallel. Therefore (2.9) implies that $A_{\xi}=c I$ at every point of $U$. Thus

$$
\begin{aligned}
\nabla_{X} A_{\xi} & =\nabla_{Y} A_{\xi}=0, \\
D_{X} \xi & =D_{Y} \xi=0 \text { at } x .
\end{aligned}
$$

From them, together with (2.7), we obtain $R(X, Y) J X=0$ (modulo normal component). In particular we have $g(R(X, Y) J X, X)=0$. Now our theorem follows from the following.

Lemma ([1], [5]). If $g(R(X, Y) J X, X)=0$ for every orthonormal $X, Y, J X \in T_{x}(M)$ and for every point $x$ of $M$, then $M$ is a complex space form, provided that $\operatorname{dim} M \geqq 3$.

\section{BIBLIOGRAPHY}

1. B. Y. Chen and K. Ogiue, Some characterizations of complex space forms, Duke Math. J. (to appear).

2. S. I. Goldberg, The axiom of 2-spheres in Kaehler geometry (to appear). 
3. D. Leung and $\mathrm{K}$. Nomizu, The axiom of spheres in Riemannian geometry, J. Differential Geometry 5 (1971), 487-489. MR 44 \#7472.

4. K. Yano and I. Mogi, On real representation of Kaehlerian manifolds, Ann. of Math. (2) 61 (1955), 170-189. MR 16, 859.

5. K. Ogiue, On invariant immersions, Ann. Math. Pura Appl. (4) 80 (1968), 387397. MR 39 \#6207.

Department of Mathematics, Tokyo Gakugei University, Koganeishi, Tokyo, JAPAN 